GEORGETOWN SCIENTIFIC RESEARCH JOURNAL
Volume One Edition One February 2021

\title{
Refractory Epilepsy: Mechanisms of Pharmacoresistance
}

Ariel Le, Makenzie Thomas, Brady Stallman, Kathryn

Meadows, Vidya Bhargava 


\title{
Refractory Epilepsy: Mechanisms of Pharmacoresistance
}

\author{
Ariel Le, Makenzie Thomas, Brady Stallman, Kathryn Meadows, and Vidya Bhargava \\ Department of Human Science, Georgetown University School of Nursing and Health Studies, \\ Washington D.C., United States of America \\ E-mail: aq13@georgetown.edu, mst82@georgetown.edu, bes104@georgetown.edu, \\ kgm65@georgetown.edu, vdb29@georgetown.edu
}

\begin{abstract}
Refractory or drug-resistant epilepsy is a complex and debilitating disorder that impacts over onethird of people diagnosed with epilepsy. Many studies have suggested a variety of possible hypotheses for drug-resistant epilepsy, including the degeneration of neural networks, alterations of anti-epileptic drug (AED) targets, intrinsic severity/frequency of seizures, and genetic predisposition to pharmacoresistance. However, extensive research suggests that the overexpression of efflux protein transporters in brain tissue is the most viable hypothesis. Specifically, the overexpression of $\mathrm{P}$-glycoproteins $(\mathrm{P}$-gps) at the blood brain barrier proves the most compelling mechanism to discuss further. Studying the mechanisms of these transporters provides critical insight for new ways to combat pharmacoresistance. Thus, this review evaluates the co-administration of $\mathrm{P}$-gp inhibitors with AEDs as a promising, yet relatively unexplored, treatment option for refractory epilepsy. This review specifically considers Tariquidar (TQD) the most promising $\mathrm{P}$-gp inhibitor for refractory epilepsy treatment. This work aims to evaluate the role of $\mathrm{P}$ gp overexpression in refractory epilepsy, consolidate current research about potential treatment options, and identify discrepancies or gaps in the literature related to $\mathrm{P}$-gp inhibitory treatments for refractory epilepsy. It was concluded that, as a result of increased drug efflux processes at the blood brain barrier, overexpression of $\mathrm{P}$-gp is the leading cause of pharmacoresistance. By inhibiting the activity of these proteins with the drug Tariquidar, an effective treatment for refractory epilepsy may become a reality.
\end{abstract}

Keywords: refractory epilepsy, pharmacoresistance, overexpression, P-glycoprotein, Tariquidar 


\section{Introduction}

Epilepsy is a chronic neurological disorder characterized by spontaneous, repeated, and unprovoked seizures resulting from abnormal electrical connections between neurons in the brain. ${ }^{1}$ Characterized by spontaneous electrical disturbances in the brain, these seizures often result in physical manifestations such as convulsion, loss of consciousness, sensory disturbances, or uncontrolled movements. ${ }^{2}$ Epilepsy encompasses many different types of seizure disorders that are classified according to relative regions of brain dysfunction, types of seizures present, and varying degrees of causal explanation. Clinical diagnosis of epilepsy requires at least two isolated seizures unrelated to brain injury, drug usage, metabolic disorders, or acute systemic dysfunction. ${ }^{3}$

Impacting 2.2 million people in the United States and over 65 million people worldwide, epilepsy is the most common neurological disorder in the world. It accounts for $0.5 \%$ of the global burden of disease, a measure that considers the number of years of life lost to premature death, illness, and disability. While this condition is prevalent across all age demographics, it disproportionately affects individuals in early childhood or late adulthood. ${ }^{3}$ People with epilepsy are up to three times more likely to experience premature death compared to the general population. 1 in every 1,000 adults with epilepsy suffer from sudden unexpected death in epilepsy (SUDEP) every year, and those with drugresistant forms are at increased risk of SUDEP. In the United States alone, over 150,000 new cases of epilepsy are diagnosed annually and one in 26 individuals are projected to develop epilepsy over the course of their lifetimes. Direct annual medical care costs of epilepsy in the United States total $\$ 9.6$ billion and contribute significant financial burden to sufferers. ${ }^{3}$ Along with the difficulties associated with seizures and other related symptoms, people living with epilepsy are often faced with challenges accessing high-quality healthcare, navigating treatment options, and dealing with public misunderstanding of the disease. In many areas across the globe, people with epilepsy also experience reduced access to educational and vocational opportunities as a result of stigma and discrimination associated with the disease. ${ }^{2}$ This emphasizes the need to find treatments or cures for epilepsy. In doing so, the detrimental impacts of the condition and the socioeconomic disparities associated with it may be relieved.

While there are numerous therapeutic options available to treat epilepsy-such as antiepileptic drugs (AEDs), surgical intervention, nerve stimulation, or specialized diets - treatment methods are often ineffective. Over one-third of people diagnosed with epilepsy will develop refractory (drug-resistant) epilepsy as a result of pharmacoresistance, the inability to control seizure activity through AED therapy. ${ }^{4}$ This form of epilepsy is clinically diagnosed after two trials of AEDs fail to manage or cease seizure activity. While about $50 \%$ of newly diagnosed epileptic patients obtain full seizure control within the first AED treatment and $13 \%$ after switching to a second AED treatment, $20-25 \%$ of total patients still do not see improvement after a single AED or a combination of AEDs. ${ }^{5}$ Management of refractory epilepsy is particularly challenging because there is little conclusive evidence about the causes or mechanisms of pharmacoresistance, and individualized treatment plans are necessary due to different underlying disease mechanisms. ${ }^{6}$ Some possible causes for drug-resistance may include environmental factors, including trauma and prior drug exposure, or genetic predispositions that impact the degree of absorption, metabolism, and uptake of AEDs at the blood brain barrier.

Most of the current research on the mechanisms of refractory epilepsy suggests that drug-resistance in epilepsy is caused by increased activity and expression of multidrug efflux transporter proteins in the ATP-binding cassette $(\mathrm{ABC})$ protein transporter family. ${ }^{7}$ There are three major types of $\mathrm{ABC}$ transporters that play a 
significant role in the failure of AEDs to control refractory epilepsy seizures: permeabilityglycoproteins (p-glycoproteins or P-gps), multidrug resistant-associated proteins (MRPs), or breast cancer resistant proteins (BCRPs). ${ }^{7}$ These active transporters use the energy of ATP through ATP hydrolysis to allow for the movement of molecules across a membrane, although $\mathrm{ABC}$ transporters specifically use an alternate access mechanism, which entails switching between "inward- and outward-facing states" at the membrane which, in turn, alternates whether the ligand-binding site is exposed inside or outside the membrane. ${ }^{8}$ Many studies suggest this group of $\mathrm{ABC}$ transporters and their related efflux mechanisms to be the major cause of pharmacoresistance in refractory epilepsy, and the most compelling evidence suggests that the overexpression, or increased expression, of Pglycoprotein transporters most significantly reduces uptake of AEDs at the blood brain barrier. ${ }^{79}$ Additional studies have shown that the inhibition of these particular P-gp transporters can reduce efflux transporter mechanisms and, subsequently, can potentially eliminate pharmacoresistance altogether.

In essence, the overexpression of $\mathrm{P}$ glycoproteins in the brain leading to the reduced uptake of AEDs at the blood brain barrier is the most promising target for the treatment of refractory epilepsy.

\section{The Transporter Hypothesis in Refractory Epilepsy}

Many studies have worked to evaluate various theories regarding the potential mechanisms of pharmacoresistance in refractory epilepsy, although there is a general consensus that protein transporters have the most influence over refractoriness. This is especially noted in a comprehensive review focused on drug-resistant epilepsy that evaluated a wide variety of studies on refractory epilepsy and summarized the potential hypotheses for mechanisms of pharmacoresistance. ${ }^{9}$ Tang et al., 2017 suggested that the drug-resistance of refractory epilepsy may come from seizure-induced neural damage that prevents anti-epilepsy drugs from accessing their neuronal targets. Another collection of studies included in the same review suggested that pharmacoresistance is inherent to the severity of epilepsy. ${ }^{8}$ While many of these hypotheses are biologically plausible, there is a significant lack of evidence, association, consistency, and specificity in the literature to demonstrate their validity.

However, one hypothesis from the review stood out as the most promising mechanism for refractory epilepsy: the transporter hypothesis. The transporter hypothesis suggests that the overexpression of efflux transporter proteins in the brain tissue of epileptic patients causes drug resistance by decreasing the permeability of the blood brain barrier for AEDs. ${ }^{10}$ There are different types of protein transporters that carry out this mechanism of pharmacoresistance: $\mathrm{P}$-gps, MRPs, and BRCs, which are all part of the $\mathrm{ABC}$ transporter family. ${ }^{7,9}$ Out of these transporters, the overexpression of $\mathrm{P}$-gp is most prominently associated with pharmacoresistance, as increased expression of $\mathrm{P}$-gp in the brain tissue of refractory epilepsy patients had a low AED response. ${ }^{9}$ The overexpression of MRPs shows similar promise as a potential mechanism of refractory epilepsy, but they have not been extensively studied with controls to further prove their association. While BCRPs perform a similar role in pharmacoresistance, it has been suggested that these transporters have better therapeutic potential for cancer rather than refractory epilepsy. ${ }^{9}$ Ultimately, based on the current findings and evidence, it can be deduced that the clearance of AEDs by $\mathrm{P}$-gps shows the most promise as a mechanism of refractory epilepsy. Thus, resources should be allocated towards exploring these proteins and investigating them as a target for future treatment.

An earlier review from 2011, which elaborated on each type of transporter and their mechanisms at the $\mathrm{BBB}$, detailed the inhibition of $\mathrm{P}$-gp expression as a potential treatment option. ${ }^{10}$ To build upon these analyses, this review focuses on P-gp overexpression, its role in 
refractory epilepsy, and potential treatments that target these mechanisms.

\section{The Overexpression and Mechanisms of P- glycoproteins in Refractory Epilepsy}

As previously mentioned, current data shows that about one-third of epilepsy patients do not respond to most types of AEDs, and it has been hypothesized in recent years that one potential mechanism for the resistance to AEDs is the overexpression of $\mathrm{P}-g \mathrm{p}$ in the blood-brain barrier (BBB) ${ }^{11}$ As ABC transporters, $\mathrm{P}$-gps use ATP hydrolysis to allow for efflux of molecules using the aforementioned alternate access mechanism. ${ }^{8,12}$ More specifically, $\mathrm{P}$-gps are exporters, so they actively work towards moving molecules outside of the cell rather than inside, so it is clear that the overexpression of these exporters are bound to lead to decreased AED concentration. ${ }^{8}$

These protein transporters can exist in large numbers in a variety of locations in the body such as the intestines, liver, kidneys, and the BBB. The protein is likely expressed in these organs to serve as a defense mechanism against foreign substances. ${ }^{12,13}$ For example, this protein exists in the intestinal epithelium, where it pumps toxins back into the intestinal lumen. P-gps can play an integral role in the effluxion, or clearance, of chronic disease drugs. Specifically, $\mathrm{P}-g \mathrm{p}$ is a key efflux protein of the $\mathrm{BBB}$ that actively transports large amounts of lipophilic drugs, including AEDs, out of the BBB membrane and back into the bloodstream, therefore resulting in pharmacoresistance to therapeutic medications intended to target the brain. ${ }^{11,12}$

There have been many clinical studies that demonstrated how the decreased concentration of $\mathrm{AED}$ s can result from $\mathrm{P}$-gp overexpression. In one case study, surgery was conducted on a patient with partial refractory epilepsy to alleviate epileptic symptoms. Prior to this study, the patient was treated with many different AEDs and doses with no measured success in controlling the patient's symptoms. ${ }^{5}$ Prior to surgery, researchers collected and measured AED blood levels in the patient's serum samples for 25 days before each administered dose of AEDs to observe the patient's AED concentration in the blood. It was found that all types of the AEDs that the patient received were observed in subtherapeutic levels in a significant portion of the serum samples collected. This indicates very low AED concentration throughout the blood. During the surgery, some samples of brain tissue were collected for immunohistochemical analysis to determine the cause of these subtherapeutic AED levels in the blood. From this analysis, it was found that the patient had high brain expression of $\mathrm{P}$ gps in not only endothelial cells of the BBB but also in astrocytes and neurons. This overexpression of $\mathrm{P}$-gps in regions where drug elimination actively takes place suggests that the protein plays an integral role in the efflux and clearance of AEDs from the brain. ${ }^{5}$ This study is consistent with other case studies on patients with refractory epilepsy.

In a rat model of epilepsy, Pgp overexpression was assessed following the administration of an anti-seizure medication, phenobarbital. Immunohistochemical analysis showed a striking overexpression of $\mathrm{P}-\mathrm{gp}$ in the limbic brain regions of the rats that did not respond to the medication. ${ }^{13}$ In particular, overexpression of $\mathrm{P}$-gp could be narrowed down to the brain capillary endothelial cells of the BBB. ${ }^{13}$ These findings were consistent with the case study mentioned above, suggesting an association between P-gp overexpression and AED response. Other studies on BCRPs and MRPs have not been able to fully conclude these same associations with the AED response, suggesting that $\mathrm{P}$-gps are the most promising candidates for a therapeutic approach.

Other experiments focused on looking at the specific mechanism of $\mathrm{P}$-gps with specific substrates. For example, one study conducted the brain-to-plasma difference of 10-OHCBZ, an active metabolite, in refractory epilepsy patients who did not respond to the AED treatment Oxcarbazepine. ${ }^{14}$ In this study, surgery was conducted to alleviate the epileptic condition of the patients, during which researchers intraoperatively measured $10-\mathrm{OHCBZ}$ levels to 
determine if it is a substrate of $\mathrm{P}$-gp and to understand the relationship between its brain-toplasma concentration ratio and the levels of expression of $\mathrm{P}$-gp. The results showed that 10OHCBZ acts as a substrate for $\mathrm{P}-\mathrm{gp}$, and the concentration of $10-\mathrm{OHCBZ}$ in the brain was not correlated to the plasma levels of the patients. However, a significant inverse linear relationship was found between the levels of expression of $\mathrm{P}$ $\mathrm{gp}$ and the brain-to-plasma concentration ratio of 10-OHCBZ. ${ }^{14}$ This inverse relationship showed that higher levels of $\mathrm{P}-\mathrm{gp}$ resulted in lower levels of the active metabolite past the $\mathrm{BBB}$, providing evidence of the hypothesized $\mathrm{P}$-gp mechanisms. The conclusions of $10-\mathrm{OHCBZ}$ apply to AEDs because the data showed that higher expressions of P-gp lead to lower expressions of 10-OHCBZ, and intracellular levels of the metabolite were increased when XR9576, a P-gp inhibitor, was introduced. However, these conclusions are not necessarily reliable due to the lack of control brain tissue from drug-responding patients. Furthermore, in-vitro studies do not necessarily confirm or reflect these mechanisms as would invivo models for refractory epilepsy. ${ }^{13}$ The observational nature of the previous study is useful in developing a base for understanding the functions of $\mathrm{P}$-gp and developing hypotheses, but in-vivo studies are needed to test and prove these hypotheses. Nevertheless, this pilot study still provided some fundamental insight into the mechanisms of $\mathrm{P}$-gp overexpression in pharmacoresistance and demonstrated how substrates of P-gp, including AEDs, have difficulty crossing the $\mathrm{BBB}$ and reaching the brain if there is an overexpression of these transporters.

An in-vivo study by Van Vliet et al. measured the levels of phenytoin (PHT), a common AED used to control seizures, in different brain regions of both epileptic and nonepileptic rats. ${ }^{15}$ The researchers wanted to compare PHT levels in regions of the brain with an overexpression of $\mathrm{P}$-gp, such as the parahippocampal cortex and the temporal hippocampus, to PHT levels in brain regions with lower P-gp expression. The concentration of PHT in regions with overexpression of $\mathrm{P}-\mathrm{gp}$ was significantly reduced compared to regions with lower P-gp expression. These results were, once again, consistent with the earlier findings, demonstrating the relationship between increased $\mathrm{P}$-gp expression and decreased levels of AED in the brain. ${ }^{15}$ Overall, it has been established, through studies using different AED substrates and refractory epilepsy models, that the overexpression of $\mathrm{P}$-gp efflux transporters leads to decreased concentration of AEDs in the brain and, consequently, pharmacoresistance. Some of these studies have also suggested this mechanism as a potential therapeutic target, specifically with the use of $\mathrm{P}$-gp inhibitors. ${ }^{15,16}$ Together, the findings of these past works suggest promising research implications for $\mathrm{P}$-gps as future treatment targets, which will be discussed in the next section.

\section{Improving Standard of Care for Refractory Epilepsy}

\subsection{Significance of $P_{-} g p$ Inbibitors in Reducing Pharmacoresistance}

Although overexpression of $\mathrm{P}$-gps has been identified as a highly probable mechanism of drug resistance in epilepsy, treatment methods have yet to significantly account for this new knowledge. A promising yet relatively unexplored treatment option lies in therapeutics that target the activity of $\mathrm{P}$-gps. $\mathrm{P}$-gp inhibitors are a specialized class of substances that block or bypass the efflux activity of $\mathrm{P}$-gps at the $\mathrm{BBB} .{ }^{16}$ In regards to AEDs specifically, these inhibitors work to prevent the efflux of AEDs from endothelial cells of the blood brain barrier(Figure 1), thus allowing for increased therapeutic effect. These inhibitors function to either block $\mathrm{P}$-gp binding sites, reduce ATP hydrolysis by $\mathrm{P}-\mathrm{gp}$, or weaken cell membrane lipids. ${ }^{16}$ The previously mentioned in-vitro study using 10-OHCBZ to observe the effect of $\mathrm{P}$ gps on intracellular drug concentration briefly introduced the use of $\mathrm{P}$-gp inhibitors, which resulted in increased intracellular drug concentration. ${ }^{14}$ This previous work was able to provide the fundamental insight needed to suggest that concurrent administration of AED substrates with P-gp inhibitors may increase drug 


\section{https://doi.org/10.48091/UWYG8998}

bioavailability or uptake at the $\mathrm{BBB}$ and result in an improved therapeutic effect. ${ }^{14,16}$

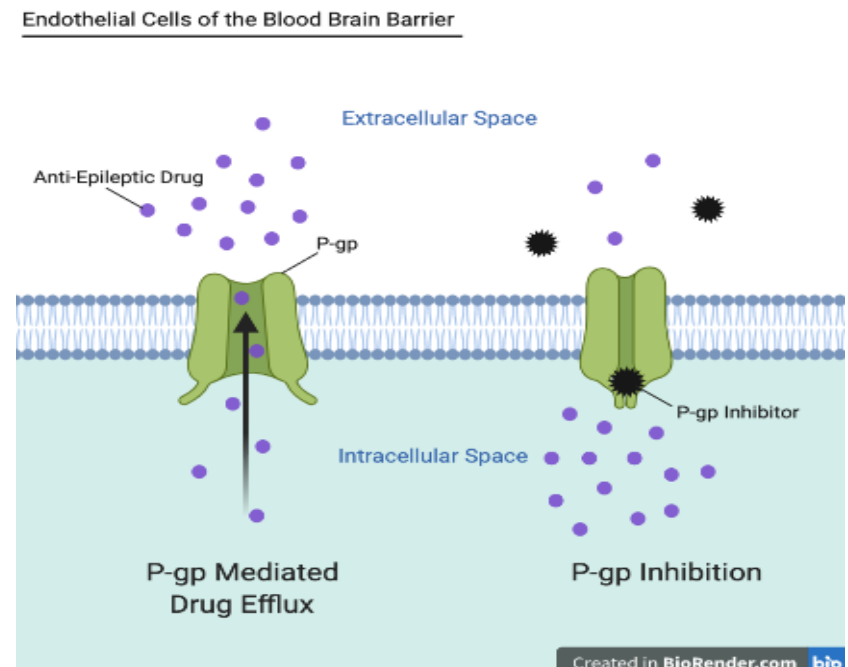

Figure 1. P-gp Inhibition to Prevent AED Efflux at the Blood Brain Barrier ${ }^{1}$

Table 1. Classification and Limitations of P-g Inhibitors

\begin{tabular}{|l|l|l|l|l|}
\hline Generation & Specificit & Limitations & Advantages & Examples \\
\hline $\begin{array}{l}\text { First } \\
\text { Generation }\end{array}$ & Low & $\begin{array}{l}\text {-High toxicity ins be administered in low } \\
\text {-Must } \\
\text { doses serum concentrations } \\
\text {-High serum } \\
\text {-Wide range of substrate } \\
\text { capabilities }\end{array}$ & $\begin{array}{l}\text {-Most pharmacologically } \\
\text {-Significant research } \\
\text { accomplished }\end{array}$ & Verapamil \\
\hline $\begin{array}{l}\text { Second } \\
\text { Generation }\end{array}$ & Medium & $\begin{array}{l}\text {-Inhibitory effect of unintended } \\
\text { enzymes and other ABC } \\
\text { transporters }\end{array}$ & -Greater affinity of P-gp & Valspodar \\
\hline $\begin{array}{l}\text { Third } \\
\text { Generation }\end{array}$ & High & $\begin{array}{l}\text {-Many still in developmental } \\
\text { stage/not ready for clinical use }\end{array}$ & $\begin{array}{l}\text {-Low toxicity } \\
\text {-Can target specific } \\
\text { locations }\end{array}$ & Tariquidar \\
\hline
\end{tabular}


Three distinct generations of Pgp inhibitors that perform similar functions have been identified (Table 1). First-generation inhibitors are substances originally developed to treat other conditions that were found to also successfully inhibit P-gps. First generation inhibitors are the most pharmacologically active, but they pose potential limitations in dosage due to their latent toxicity, unusually high serum concentrations, and ability to substrate with a wide range of unintended, biologically beneficial transporters and enzymes. ${ }^{16}$ Second-generation inhibitors do not hold the same range of pharmacological ability as the first-generation, but they do possess a greater affinity for P-gps, allowing for more specific targeting in the body. However, second-generation inhibitors are limited in clinical use by their tendency to inhibit certain enzymatic activities and other $\mathrm{ABC}$ transporters that lead to complicated drug-drug interactions. ${ }^{16}$ Most third-generation inhibitors are still in the clinical development phase and aim to act with high $\mathrm{P}$-gp specificity and low toxicity. While all three generations of $\mathrm{P}$-gp inhibitors face potential restrictions in their use, they also offer promising properties as a clinical treatment for refractory epilepsy.

\subsection{Tariquidar as a Promising Therapeutic Option}

In identifying $\mathrm{P}$-gp inhibition as a potential therapeutic option for refractory epilepsy, it is important to consider the specific functions and locations of these inhibitors. P-gp inhibitors may specifically target overexpression in the intestines, liver, brain, and kidneys or may only play a pharmacological role in increasing the uptake of certain drugs. ${ }^{17}$ Two such Pgp inhibitors, Tariquidar (TQD) and Verapamil, have been identified as potential treatments for refractory epilepsy due to their high specificity in targeting the blood brain barrier.

Many studies have provided evidence to indicate that Tariquidar, or TQD, is a promising selective inhibitor of $\mathrm{P}$-gp that may offer a solution to refractory epilepsy. As previously tested by Van
Vliet, E. A. et al., TQD shows great potential in counteracting pharmacoresistance of AEDs resulting from the overexpression of $\mathrm{P}$ gps. ${ }^{15} \mathrm{TQD}$ is a third-generation $\mathrm{P}$-gp inhibitor known for its high selectivity, long active period, and advantageous oral administration and bioavailability. ${ }^{17}$ Since its development, this exceptionally potent inhibitor has been considered one of the best ways to reduce the effects of $\mathrm{P}$ $\mathrm{gp}$ overexpression. The oral intake of drugs such as TQD is often the most convenient and safest route of drug administration and, following the administration of TQD specifically, has shown to have a relatively strong bioavailability. TQD also acts as a non-competitive inhibitor to reduce the ATP hydrolyzing activity of $\mathrm{P}$-gps. This activity facilitates the transport of substances out of cells; thus, TQD inhibits such behavior that is key to pharmacoresistance. ${ }^{17}$ One study demonstrated the high potency of TQD as a P-gp inhibitor and discovered that the administration of one micromolar of TQD decreased the ATPase activity of $\mathrm{P}-\mathrm{gp}$ by over $50 \% .{ }^{18}$ While TQD has mainly been studied as a modulator of reversing multidrug resistance in cancer, current evidence has also identified TQD as a potential modulator of pharmacoresistance in epilepsy. ${ }^{12}$

Many studies have demonstrated the inhibitory effect of TQD on P-gp overexpression that contributes to refractory epilepsy. Building upon their previous work measuring AED uptake in brain regions with $\mathrm{P}$-gp overexpression, Van Vliet, E. A. et al. continued an investigation into the anticonvulsant effects of phenytoin (PHT) before and after administration of TQD. After $\mathrm{co}^{-}$ administering PHT and TQD to rats with frequent daily seizures, researchers analyzed brainto-plasma concentration ratio measurements of PHT. ${ }^{19}$ The researchers also recorded the frequency of seizures to determine any observable changes in the epileptic activity of the rats when treated with TQD. The results showed that, when combined with TQD, PHT levels in the rats remained within the therapeutic range in the blood for an additional 8 hours compared to PHT alone. 
Additionally, overall seizure activity was reduced in rats treated with both drugs. In the control group, PHT treatment alone did not significantly decrease seizure activity until day 13 . Conversely, the experimental group of PHT co-administered with TQD showed reduction in seizures during the first 3 days of treatment. This experiment concluded that the administration of TQD allowed for an increased uptake of PHT at the $\mathrm{BBB}$, inhibiting the mechanism of $\mathrm{P}$-gps in chronic epileptic rats and, thus, dramatically reducing the frequency of seizures. This analysis demonstrates TQD's ability to significantly reduce $\mathrm{P}$-gp activity and provides a promising approach for improving the standard of care for refractory epilepsy.

A second major study showed consistent findings on the effects of TQD in reducing Pgp pharmacoresistance when co-administered with phenobarbital (PB), a drug widely used for its anti-epileptic effect. ${ }^{20}$ In this study, researchers used EEG monitoring and blood sampling to analyze seizure activity and drug concentration in plasma. The study used a rat model of temporal lobe epilepsy with pre-established drug-resistance to phenobarbital. In one trial, co-administration of TQD and PB completely eliminated seizure activity in four nonrespondent rats and reduced seizures by over $90 \%$ in the remaining two nonrespondent rats compared to only a $24 \%$ reduction in the control group receiving $\mathrm{PB}$ alone. In a remarkable example, one rat went from experiencing an extensive number of seizures per day before treatment to becoming completely seizure free with the co-administration of TQD and $\mathrm{PB}$. This study concluded that the $\mathrm{co}^{-}$ administration of TQD with $\mathrm{PB}$ completely restored the anti-epileptic effect of the drug in pharmacoresistant rat models of temporal lobe epilepsy. ${ }^{19}$ Another comparable study conducted using human subjects discovered a 2.5 fold increase in AED uptake at the BBB when co-administered with TQD. ${ }^{21}$ Combined with the previous study, these results provide convincing evidence that the $\mathrm{P}$-gp inhibitor, TQD, counteracts drug-resistance in epileptic rats and could act as an effective treatment method to improve the standard of care in refractory epilepsy.

In a single case study, a patient was administered Verapamil in conjunction with an AED proven to be a reliable treatment method. ${ }^{22}$ With the combination of Verapamil and the AED, the patient was able to double the time between hospitalizations for seizures, demonstrating a significant improvement. Even so, researchers determined that it was impossible to isolate the cause for the improvement, as it could have resulted from Verapamil, the viable AED, the placebo effect, or any combination of the three. Only further research in a controlled setting would determine if Verapamil is a reliable $\mathrm{P}$-gp inhibitor and treatment for refractory epilepsy. Moreover, even if researchers could determine that Verapamil was the cause for improvement, they could not determine the mechanism by which Verapamil acted. With only this one major study providing inconclusive evidence, Verapamil lacks the support needed to establish its efficacy as a P-gp inhibitor and positions Tariquidar as the better therapeutic option. $^{22}$

\subsection{Limitations of TQD}

While many developments have been made in studying refractory epilepsy, significant uncertainty must be accounted for to better understand treatment options, especially in relation to TQD. Although TQD shows the most promise as a treatment for refractory epilepsy, there are limitations that should be considered. Recent TQD studies have demonstrated significant adverse events. The aforementioned study by Van Vliet E. A. et al. reported significant abdominal pain in the TQD treatment group, resulting in a dissolution of the trial. While examining the potential application of TQD for multidrug resistance in cancer, research was discontinued after $41 \%$ of subjects reported adverse effects to the drug. ${ }^{23}$ Researchers in one study concluded that administration of 
TQD was not associated with enhanced toxicity or altered blood plasma pharmacokinetics as in the case of several other $\mathrm{P}$-gp inhibitors, yet these results have not been replicated in similar studies nor demonstrate consistency with other findings. ${ }^{20}$ Two studies revealed that, despite the significant improvement in seizure activity during the first few days of TQD and AED coadministration, TQD tolerance eventually developed through unknown mechanisms. ${ }^{19,21}$ These results implicate the need for further exploration of the pharmacology of TQD in order to reduce tolerance to the drug. TQD could potentially lead to toxicity in organs other than the brain, such as the intestines, liver, and kidneys by inhibiting the efflux effect of $\mathrm{P}$-gps expressed in those organs as well. ${ }^{17}$ More knowledge on the adverse events of TQD is necessary to ensure its safety in the clinical setting. Additionally, since current research predominantly considers the effects of TQD for two types of AEDs (phenobarbital and phenytoin), further investigation on whether these effects can be extrapolated to a broader range of AEDs would provide a more comprehensive treatment method for refractory epilepsy.

\section{Conclusion}

Refractory epilepsy is a complex, multifaceted disease that impacts millions of people worldwide. The drug-resistant nature of this condition poses unique challenges for physicians attempting to provide therapeutic care to patients and establishes an urgency for researchers to determine its mechanisms. While many hypotheses attempt to explain the mechanisms of refractory epilepsy, the overexpression of P-glycoprotein (P-gps) transporters in endothelial cells at the blood brain barrier best accounts for pharmacoresistance. Across the numerous studies examined within this review, a clear relationship was identified between the overexpression of $\mathrm{P}$-glycoproteins in epileptic brain tissue and reduced uptake of antiepileptic drugs (AEDs) at the blood brain barrier. The consistent and replicated association between $\mathrm{P}$ gp overexpression and reduced AED concentration demonstrates that, as a result of the drug efflux process, $\mathrm{P}$-gp overexpression is the primary mechanism of decreased drug efficacy in refractory epilepsy patients.

With P-gp overexpression identified as the lead cause of refractory epilepsy, the focus shifts towards improving the standard of care for refractory epilepsy patients through the proposed treatment method of $\mathrm{P}$-gp inhibition. This treatment method suggests that the $\mathrm{co}^{-}$ administration of an AED and P-gp inhibitor reduces the efflux effects of $\mathrm{P}$-gp and allows for increased AED uptake at the blood brain barrier, thereby increasing the therapeutic effect of the drug. Two P-gp inhibitors, Tariquidar (TQD) and Verapamil, have been identified as potential treatment options. However, due to the lack of evidence supporting Verapamil's efficacy, it has been determined that TQD poses the most promise as an effective treatment option for refractory epilepsy. Many studies examined within this review showed that when co-administered with an established AED, TQD led to increased AED concentration in brain tissue and, thus, greater drug efficacy. Despite these promising findings, Tariquidar still poses limitations that warrant further investigation. Many studies have shown that TQD may cause adverse events such as tolerance and toxicity. Additionally, very few studies using human subjects have been conducted on TQD inhibitors and their effect with various AEDs. With these limitations and gaps in knowledge, the need for more clinical trials specifically investigating the effects of TQD and other P-gp inhibitors such as Verapamil is clear. In addition, future studies should be conducted to determine the mechanisms of TQD tolerance and toxicity in order to improve the safety and efficacy of TQD. Such findings may affirm the ability of $\mathrm{P}$-gp inhibitorto overcome pharmacoresistance and finally providean effective therapeutic strategy for refractory epilepsy. 


\section{References}

1. Epilepsy. Mayo Clinic. https://www.mayoclinic.org/diseasesconditions/epilepsy/symptoms-causes/syc20350093. Published May 5, 2020. Accessed December 15, 2020.

2. Epilepsy. World Health Organization. https://www.who.int/news-room/factsheets/detail/epilepsy. Published June 20, 2019. Accessed December 15, 2020.

3. Sirven JI. Epilepsy: A Spectrum Disorder. Cold Spring Harbor Perspectives in Medicine. 2015;5(9). doi:10.1101/cshperspect.a022848

4. Wirrell E, ed. Drug-Resistant Epilepsy. Epilepsy Foundation. https://www.epilepsy.com/learn/drugresistant-epilepsy. Published October 5, 2020. Accessed December 15, 2020.

5. Lazarowski A, Massaro M, Schteinschnaider A, Intruvini S, Sevlever G, Rabinowicz A. Neuronal MDR-1 Gene Expression and Persistent Low Levels of Anticonvulsants in a Child with Refractory Epilepsy. Therapeutic Drug Monitoring. 2004;26(1):44-46. doi:10.1097/00007691-200402000-00010

6. French JA. Refractory Epilepsy: Clinical Overview. Epilepsia. 2007;48(s1):3-7. doi:10.1111/j.1528-1167.2007.00992.x

7. Lazarowski A, Czornyj L, Lubienieki F, Girardi E, Vazquez S, D'giano C. ABC Transporters during Epilepsy and Mechanisms Underlying Multidrug Resistance in Refractory Epilepsy. Epilepsia. 2007;48(s5):140-149. doi:10.1111/j.15281167.2007.01302.x

8. Beis K. Structural basis for the mechanism of ABC transporters. Biochemical Society Transactions. 2015;43(5):889-893. doi:10.1042/bst20150047
9. Tang F, Hartz AMS, Bauer B. Drug-

Resistant Epilepsy: Multiple Hypotheses, Few Answers. Frontiers in Neurology. 2017;8. doi:10.3389/fneur.2017.00301

10. Bauer B, Schlichtiger J, Pekcec A, M.s. A. The Blood-Brain Barrier in Epilepsy. Clinical and Genetic Aspects of Epilepsy. September 2011. doi:10.5772/21561

11. Sisodiya S. Etiology and management of refractory epilepsies. Nature Clinical Practice Neurology. 2007;3(6):320-330. doi:10.1038/ncpneuro0521

12. Löscher W, Potschka H. Blood-brain barrier active efflux transporters: ATP-binding cassette gene family. NeuroRX. 2005;2(1):8698. doi:10.1602/neurorx.2.1.86

13. Volk HA, Löscher W. Multidrug resistance in epilepsy: rats with drug-resistant seizures exhibit enhanced brain expression of $\mathrm{P}$ glycoprotein compared with rats with drugresponsive seizures. Brain. 2005;128(6):13581368. doi:10.1093/brain/awh437

14. Marchi N, Guiso G, Rizzi M, et al. A Pilot Study on Brain-to-Plasma Partition of 10,11Dyhydro-10-hydroxy-5Hdibenzo(b,f)azepine-5-carboxamide and MDR1 Brain Expression in Epilepsy Patients Not Responding to Oxcarbazepine. Epilepsia. 2005;46(10):1613-1619. doi:10.1111/j.15281167.2005.00265.x

15. Vliet EAV, Schaik RV, Edelbroek PM, et al. Region-Specific Overexpression of Pglycoprotein at the Blood-Brain Barrier Affects Brain Uptake of Phenytoin in Epileptic Rats. Journal of Pharmacology and Experimental Therapeutics. 2007;322(1):141147. doi:10.1124/jpet.107.121178

16. Srivalli KMR, Lakshmi PK. Overview of Pglycoprotein inhibitors: a rational outlook. Brazilian Journal of Pharmaceutical Sciences. 2012;48(3):353-367. doi:10.1590/s198482502012000300002 
17. Amin ML. P-glycoprotein Inhibition for Optimal Drug Delivery. Drug Target Insights. 2013;7:27-34. doi:10.4137/dti.s125192004;38(10):16311634. doi:10.1345/aph.1e68

18. Weidner LD, Fung KL, Kannan P, et al. Tariquidar Is an Inhibitor and Not a Substrate of Human and Mouse P-glycoprotein. Drug Metabolism and Disposition. 2015;44(2):275282. doi:10.1124/dmd.115.067785

19. Vliet EAV, Schaik RV, Edelbroek PM, et al. Inhibition of the Multidrug Transporter PGlycoprotein Improves Seizure Control in Phenytoin-treated Chronic Epileptic Rats. Epilepsia. 2006;47(4):672-680. doi:10.1111/j.1528-1167.2006.00496.x

20. Brandt C, Bethmann K, Gastens AM, Löscher $\mathrm{W}$. The multidrug transporter hypothesis of drug resistance in epilepsy: Proof-of-principle in a rat model of temporal lobe epilepsy. Neurobiology of Disease. 2006;24(1):202-211. doi:10.1016/j.nbd.2006.06.014

21. Bauer M, Zeitlinger M, Karch R, et al. PgpMediated Interaction Between (R)[11C]Verapamil and Tariquidar at the Human Blood-Brain Barrier: A Comparison With Rat Data. Clinical Pharmacology \& Therapeutics. 2011;91(2):227-233. doi:10.1038/clpt.2011.217

22. Summers MA, Moore JL, Mcauley JW. Use of Verapamil as a Potential P-Glycoprotein Inhibitor in a Patient with Refractory Epilepsy. Annals of Pharmacotherapy. 2004;38(10):1631-1634. doi:10.1345/aph.1e068

23. Pusztai L, Wagner P, Ibrahim N, et al. Phase II study of tariquidar, a selective Pglycoprotein inhibitor, in patients with chemotherapy-resistant, advanced breast carcinoma. Cancer. 2005;104(4):682-691. doi:10.1002/cncr.21227 


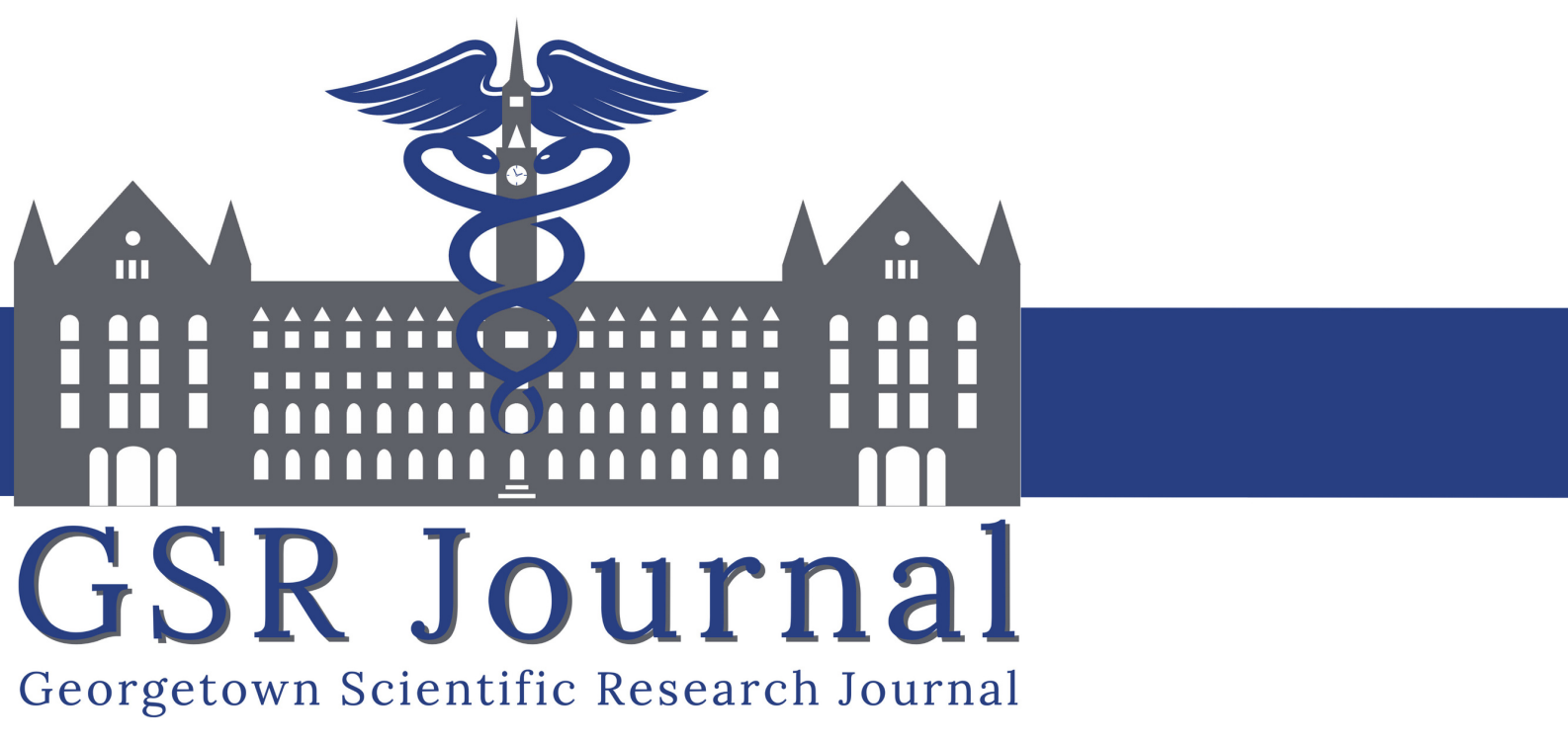

